Udo Reifner

\title{
Finanzielle Allgemeinbildung
}

Bildung als Mittel der Armutsprävention

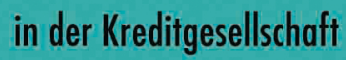

NOMOS

Verlagsgesellschaft mbH 


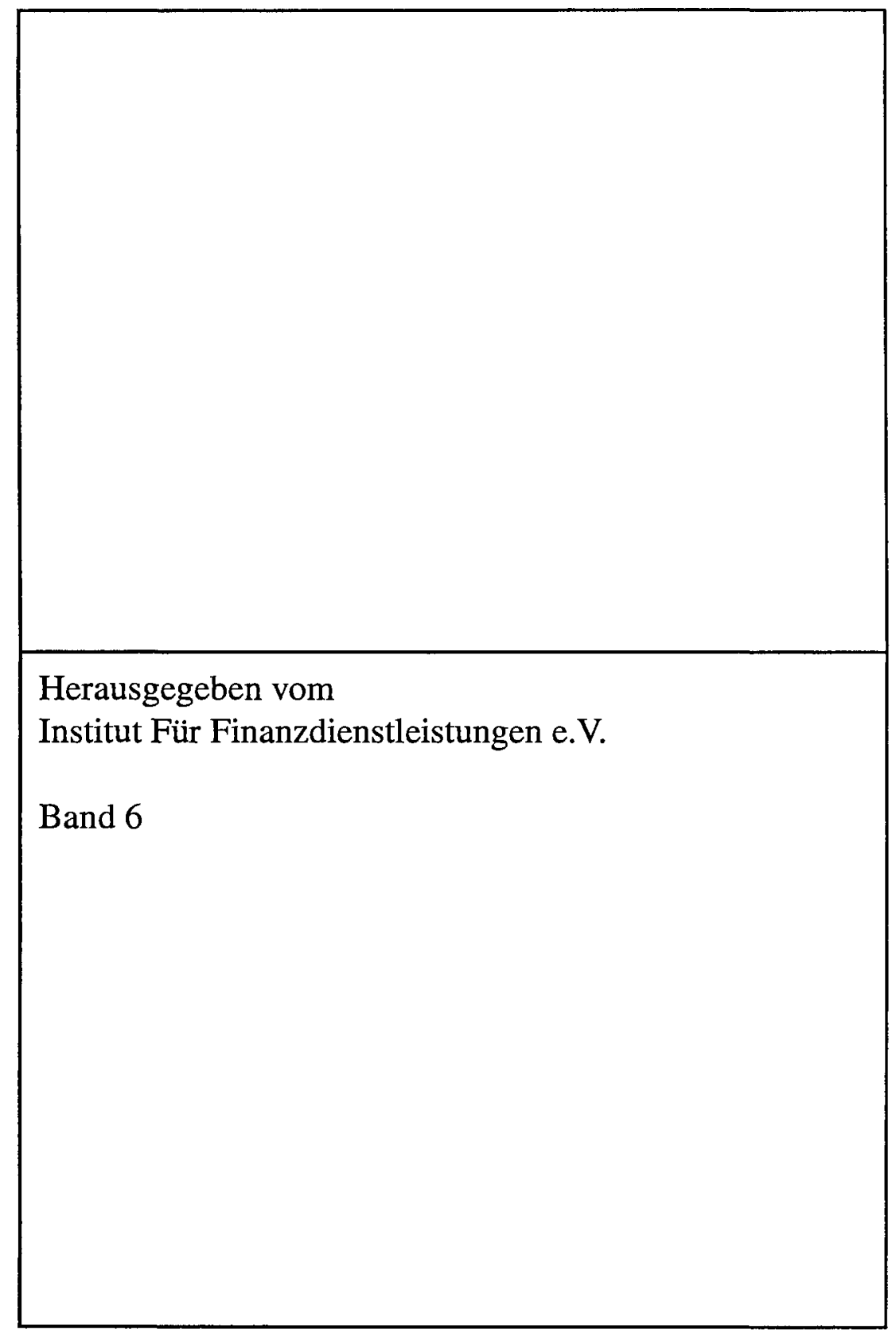


Prof. Dr. Udo Reifner

\section{Finanzielle}

\section{Allgemeinbildung}

Bildung als Mittel der Armutsprävention in der

Kreditgesellschaft

Projektabschlussbericht zur ersten Phase des vom Bundesministerium für Familie, Senioren, Frauen und Jugend unterstützten Projektes 
Bibliografische Information Der Deutschen Bibliothek

Die Deutsche Bibliothek verzeichnet diese Publikation in der Deutschen Nationalbibliografie; detaillierte bibliografische Daten sind im Internet über http://dnb.ddb.de abrufbar.

ISBN 3-7890-8362-3

1. Auflage 2003

(c) NOMOS Verlagsgesellschaft, Baden-Baden 2003, Printed in Germany. Alle Rechte, auch die des Nachdrucks von Auszügen, der photomechanischen Wiedergabe und der Übersetzung vorbehalten. Gedruckt auf alterungsbeständigem Papier. 
Die vorliegende Arbeit schließt den ersten Teil eines auf insgesamt drei Jahre angelegten Forschungsprojekts ab, der von 1999 bis 2001 im Institut für Finanzdienstleistungen mit Unterstützung des Bundesministeriums für Familie, Senioren, Frauen und Jugend durchgeführt wurde. Es sollte die Rahmenbedingungen für die Frage untersuchen, inwieweit eine neue Anstrengung in der Allgemeinbildung möglich und notwendig ist, eine veränderte Nutzung der Finanzdienstleistungen zu ermöglichen, durch die ein stabiler wirtschaftlicher Rahmen für Haushalte begünstigt bzw. Prozesse der Verarmung verhindert werden können. Das Projekt, das vom zuständigen Referat im Bundesministerium für Familie, Senioren, Frauen und Jugend aktiv und mit Engagement begleitet wurde, war für das Institut für Finanzdienstleistungen insofern Neuland, als das Institut zwar Bildungsprozesse praktisch mit gestaltet, nicht aber über Bildung geforscht hat.

Deshalb kam es wesentlich darauf an, in Auseinandersetzung mit der jeweiligen Praxis, in der Bildung, Information und Beratung stattfindet, die Kompetenz des Instituts bei der kritischen Begleitung von Finanzdienstleistungen einzubringen und dabei gleichzeitig von den Bildungskompetenzen anderer Träger zu profitieren. Hierzu dienten insbesondere auch zwei projektbegleitende Seminare, die einerseits mit Finanzberatern aus dem Bereich Financial Planning sowie der unabhängigen Investment- und Versicherungsberatung, zum anderen mit Vertretern der Schuldner- und Verbraucherberatung durchgeführt wurden. Die Zwischenergebnisse des Projektes wurden in zwei weiteren Veranstaltungen Vertretern aus Bildungsforschung, Bildungsinstitutionen sowie aus anderen, vom Bundesministerium für Familie, Senioren, Frauen und Jugend geförderten Projekten zur Armutsbekämpfung zur Diskussion gestellt. Die Projektlaufzeit war von einem dreimonatigen Aufenthalt des Verfassers an der New York University begleitet, wo Material gesammelt, Initiativen besucht und Interviews mit Banken und Community Development - Gruppen durchgeführt wurden.

Das Projekt ist durch unruhiges Fahrwasser gefahren. Eine Zeit, in der die Finanzwelt euphorische Möglichkeiten suggerierte und viele meinten, die Geschichte von Armut und Reichtum müsse neu geschrieben werden, blieb auch nicht ohne Einfluss auf die Erwartungen und Ziele des Projektes. Die Heterogenität der Projektmitarbeiter ${ }^{1}$ hat auch heterogene konzeptionelle Ansätze hervorgerufen und teilweise fruchtbare, aber gegensätzliche Standpunkte produziert: Von der Ansicht, dass das Wissen der Unterschichten ,zu wenig“ sei und daher über Schule und Medien an das Niveau einer Finanzgesellschaft herangeführt werden müsse, über einen Schwerpunkt im Umgang mit Finanzanlagen bis hin zur Auffassung, dass der bildungsmäßige Schutz der Interessen des Mittelstandes an der Teilhabe am Kapitalmarkt als Verbraucherschutz zugleich auch Armutsprävention sei, ohne dass man sich um Armut selber kümmern müsse, hat das Projekt die in ihm beschriebenen Varianten selbst durchlebt.

Der Abschlussbericht hat dann auf Materialien der Unterprojekte zurückgegriffen, gleichwohl aber eine eigene Konzeption verfolgt, für die der Verfasser die alleinige Verantwortung trägt. Nach dieser Konzeption von finanzieller Allgemeinbildung wird Bildung als „Herausbildung" von subjektiven wie objektiven Strukturen verstanden, die

1 Folgende Berufsdisziplinen waren vertreten: Wirtschaftswissenschaften, Soziologie, Rechtswissenschaften, Journalistik, Sozialpädagogik und Ökotrophologie. 
Armut verhindern helfen. Das Projekt blieb damit in dem Rahmen, den die Forschungen am IFF von jeher hatten: der Anpassung wirtschaftlicher Strukturen an die Menschen, die darin leben.

$\mathrm{Zu}$ dem Abschlussbericht hat vor allem Dagmar Hayen mit ihrer Arbeit zu den bildungstheoretischen Ansätzen unter C. III., mit ihrer Auswertung der Lehrpläne sowie der Befragung von Ministerien und Schulen unter E.II. beigetragen. Die Untersuchungen zur Beratung durch soziale Organisationen, deren Ergebnisse unter E. III. beschrieben sind, stammen von Daniela Litschke. Die Bildungsmaterialien der anbietenden Wirtschaft, auf der die Evaluation unter E.IV. beruht, hat Stefan Eisele zusammengetragen. Der Teil über die Bildung beruht auf einem Gutachten von durch Marco Habschick.

Für die Hilfe bei der Bearbeitung der vorgeschlagenen Modellprojekte habe ich schließlich Lara Louwien zu danken.

Besonderen Dank dafür, dass das Projekt mit seinen Wechselungen und veränderten Erkenntnissen nicht gescheitert, sondern wie wir glauben zu einem Ergebnis geführt worden ist, das ähnliche Diskussionen hervorbringen und bereichern könnte, haben das Bundesministerium für Familie, Senioren, Frauen und Jugend durch Herrn Ministerialrat a.D. Frank Bertsch und Herrn Albert Klein-Reinhardt mit ihren Beiträgen und ihrem Vertrauen beigetragen.

Großer Dank gebührt auch den vielen Vertretern aus Wissenschaft und Praxis, aus dem Bereich der ökonomischen Bildung, aus Schule sowie Finanz-, Verbraucher- und Schuldenberatung ebenso wie den Journalisten aus Presse und Fernsehen, die uns mit Material und Information bereitwillig geholfen, unser Anliegen unterstützt und als Gesprächspartner der jeweiligen Ergebnisse bei Evaluation und Gestaltung geholfen haben.

Seit 2002 gibt es die unter F. skizzierten Modellprojekte zur Anreicherung bestehender Bildungs-, Beratungs- und Informationsprozesse mit Elementen finanzieller Allgemeinbildung in Hamburger Schulen, bei der Schuldnerhilfe Essen e.V. und der Schuldnerhilfe Köln e.V. sowie in Kooperation mit dem NDR. Wir hoffen in diesen Projekten zeigen zu können, dass es um die Fortentwicklung vorhandener Lerninhalte und -strukturen in der Kreditgesellschaft geht. Geldgeschäfte als Dienstleistungen sind kein Spezialfach sondern in allen Lebensbereichen und damit auch in allen Lerninhalten präsent.

Hamburg, den 08.12.02 
A. Finanzielle Allgemeinbildung gegen Armut 15

I. Heraus-"Bildung" von Finanzdienstleistungen 15

II. Finanzielle und ökonomische Bildung 19

$\begin{array}{ll}\text { B. Finanzdienstleistungen und Verarmung } & 24\end{array}$

I. Armut als Prozess: von der Status- zur Verfahrensarmut 25

$\begin{array}{ll}\text { II. Armut durch Finanzdienstleistungen } & 27\end{array}$

1. Lebensnotwendigkeit von Finanzdienstleistungen 28

2. Bedrohung durch Finanzdienstleistungen $\quad 33$

a. Die Armen zahlen mehr 33

b. Die Armen erhalten weniger: Armutsprodukte 35

3. Ausschluss von Finanzdienstleistungen $\quad 36$

4. Teilhabe oder Mindeststandards $\quad 38$

III. Armutsprävention bei Finanzdienstleistungen $\quad 40$

1. Informierte Auswahl 43

2. Rechtlicher Schutz 45

3. Marktmacht 49

a. Solidarität als Verbrauchermacht 49

b. Wege zur Solidarität 53

IV. Anforderungen an finanzielle Allgemeinbildung $\quad 58$

$\begin{array}{ll}\text { C. Bildungskonzepte und finanzielle Emanzipation } & 61\end{array}$

I. Bildung als Prinzip $\quad 61$

II. Ökonomische Bildung und Financial Literacy 65

1. Ökonomische Bildung in Deutschland 65

2. Financial Literacy in den angelsächsischen Ländern 67

3. Begriff und Ziele der finanziellen Bildung $\quad 70$

III. Pädagogische Ansätze zur finanziellen Bildung $\quad 73$

1. Lehrinstanzen: Lehren wo gelernt wird 73

2. Lernziele: aktive und solidarische Verbraucher 75

3. Methodik: Lernen für konkretes Handeln 77

4. Lernmodelle: Lernen an Problemen 83

a. Modelle handlungsorientierten Lernens $\quad 83$

b. Die Zukunftswerkstatt 84

c. Die Methode der einfachen Heuristiken 87

IV.Zusammenfassung $\quad 89$ 
I. Funktionen und Zwecke

1. Zweck der Nutzung

2. Soziale Situation und Nutzungsphase $\quad 95$

$\begin{array}{ll}\text { II. Produkte und Situationen } & 97\end{array}$

1. Aufnahme von Konsumkrediten 97

2. Führen eines Girokontos 102

3. Eigenheimfinanzierung 106

4. Versicherungen 110

5. Geldanlage/Private Altersvorsorge $\quad 112$

6. Existenzgründung $\quad 115$

$\begin{array}{ll}\text { 7. Umschuldung } & 119\end{array}$

E. Die Praxis finanzieller Bildung in Deutschland 122

I. Fragestellungen, Ziele und Methoden der Erhebung 122

II. Schulunterricht in allgemeinbildenden Schulen 124

1. Erhebungsziele und -methoden 124

2. Lehrpläne, Rahmenpläne und Rahmenrichtlinien $\quad 126$

a. Die Wirtschaftsfächer $\quad 126$

b. Geld innerhalb der Wirtschaftsfächer 132

c. Zusammenfassung 139

3. Praxis des Unterrichts 140

4. Schulbücher und Unterrichtsmaterialien 144

a. Schulbuch „Überschuldung von Jugendlichen“ 145

b. Mathematik-Lehrbücher 152

(1) Allgemeines $\quad 152$

(2) Lebensfremde Beispiele $\quad 152$

(3) Problematische Zinsrechnung 153

(4) Liquidität von Anlagen $\quad 155$

(5) Kredit und Sicherheit vermischt 156

(6) Tilgungen werden außer Acht gelassen $\quad 156$

(7) Komplexität und Vereinfachung $\quad 156$

(8) Werbung 157

(9) Zusammenfassende Beurteilung 158

c. Anbietermaterialen 159

d. Schuldnerberatungsstellen und Verbraucherzentralen ${ }^{191} \quad 163$

5. Schulprojekte und Initiativen 166

6. Zusammenfassung 169

III. Weiterbildung durch soziale Organisationen $\quad 171$

$\begin{array}{ll}\text { 1. Verbraucherzentralen } & 171\end{array}$

2. Schuldnerberatungsstellen 177

3. Zusammenfassung 182 
IV. Bildungsinformation der anbietenden Wirtschaft

1. Geschäftsbanken

2. Genossenschaftsbanken

3. Sparkassen

4. Versicherungen

5. Zusammenfassung

V. Aufklärung durch die Medien

1. Themenschwerpunkte

2. Präsentationsform

3. Zielgruppen

4. Bildungsanspruch der Redaktionen

5. Trends

6. Ergebnis

VI. Finanzielle Allgemeinbildung in den USA

1. Die Kampagne zur „Finanziellen Alphabetisierung“

a. Überschuldung und Verarmung 208

b. Was ist „Financial Literacy“? 209

c. Ziele und Methoden der Financial Literacy 210

2. Financial Literacy Progranme 213

a. Financial Literacy im Gymnasium 213

b. Bankaufsicht 215

c. Geschäftsbanken 217

d. Schuldnerberatung (Credit Counseling Services) 218

3. Finanzdienstleistungen durch Alternativorganisationen 219

a. Die Organisation der gemeinnützigen Finanzentwicklungsinstitute (CDFIs)

b. Financial Literacy durch einzelne CDFIs 222

(1) Bürgerorganisationen $\quad 222$

(2) Kreditgenossenschaften 224

(3) Community Development Banks $\quad 226$

4. Zusammenfassung 227

F. Modelle zur Finanziellen Bildung 229

I. Finanzielle Allgemeinbildung in der Schuldnerberatung 230

$\begin{array}{ll}\text { 1. Projektansatz } & 230\end{array}$

2. Projektziel 231

3. Methodik 231

4. Form und Inhalt 233

5. Evaluation 234

II. Praxisorientierte finanzielle Allgemeinbildung an Schulen 234

$\begin{array}{ll}\text { 1. Projektansatz } & 234\end{array}$

2. Projektziel 235

3. Methodik 235

4. Form und Inhalte 235 
5. Reform innerhalb der Fächer 235

a. Praxissimulation in Projektwochen 236

b. Planspiel Entschuldung 236

c. Bank in der Schule 236

III. Gruppenberatungen in einer Stadtteilfiliale $\quad 237$

$\begin{array}{ll}\text { 1. Projektansatz } & 237\end{array}$

2. Projektziel 237

3. Methodik 238

4. Form und Inhalt 239

5. Evaluation 239

IV.Finanzielle Allgemeinbildung im Nachmittagsprogramm des Fernsehens $\quad 240$

1. Projektansatz 240

2. Projektziel 241

3. Methodik 241

4. Form und Inhalt 242

5. Evaluation 242

$\begin{array}{ll}\text { G. Literaturverzeichnis } & 243\end{array}$ 


\section{Abbildungsverzeichnis}

Abbildung 1: Magisches Viereck finanzieller Grundbildung

Abbildung 2: Ökonomische Bildung

Abbildung 3: Kreditzuwachs nach Kundengruppen

Abbildung 4: Konsumentenkreditvolumen

Abbildung 5: Marktregulierung und Zugang

Abbildung 6: Reflexiver Gestaltungsprozess zwischen Anbieter und Verbraucher

Abbildung 7: Lebensliquidität

Abbildung 8: Schulbuchauszug Mathematik zur Zinsrechnung

Abbildung 9: Bauspardarlehen im Schulbuch

Abbildung 10: Caritas Sozialberatung für Schuldner

Abbildung 11: Information Commerzbank

Abbildung 12: 7 Plus

Abbildung 13: Themen von Fernsehsendungen

Abbildung 14: Kreditinformation in den USA

Abbildung 15: Ausbildungsdarlehen in den USA

\section{Tabellenverzeichnis}

Tabelle 1: Girokonten

Tabelle 2: Kapitallebensversicherungen

Tabelle 3: Kredite für Privathaushalte

Tabelle 4: Bankenkonzentration in Europa

Tabelle 5: Bankfilialen in Deutschland

Tabelle 6: Mittel der Armutsprävention und Verbraucherschutz

Tabelle 7: Recht, Aufklärung, Gegenmacht

Tabelle 8: Praxisnähe und Bildungseinrichtung

Tabelle 9: Finanzdienstleistungen und Lernziele

Tabelle 10: Finanzdienstleistungen und Lernkonzepte

Tabelle 11: Lebensphasen der Produkte und Risikosituationen der Betroffenen

Tabelle 12: Auswirkungen von Ratenrückständen

Tabelle 13: Phasen und Probleme gescheiterter Baufinanzierungen

Tabelle 14: Umschuldungskarussel

Tabelle 15: Unterrichtsfächer mit Bezug zu finanzieller Bildung an den allgemeinbildenden Schulen der Sekundarstufen I und II

Tabelle 16: Arbeits- und Wirtschaftsansatz im geldbezogenen Unterricht

Tabelle 17: Geldthemen im Unterricht

Tabelle 18: Kreditkartenschulden und Jahreseinkommen 
